\title{
Auditory Working Memory: A Comparison Study in Adults with Normal Hearing and Mild to Moderate Hearing Loss
}

\author{
Rinu Annie Roy* \\ Masters in Clinical Audiology and Hearing Therapy, School of Advanced Education Research and Accreditation, University of Isabel 1, Spain
}

Submission: January 22, 2017; Published: February 15, 2018

*Corresponding author: Masters in Clinical Audiology and Hearing Therapy, School of Advanced Education Research and Accreditation, University of Isabel 1, Spain, Email: rinu.roy86@yahoo.co.in

\begin{abstract}
Working memory has a crucial role in one's effective communication, especially for the speech and language comprehension. The purpose of this study is to compare the auditory working memory capacity (AWMC) in normal hearing adults and adults with mild to moderate hearing loss using digit span tasks from WAIS- IV. This paper attempts to increase the audiologist's knowledge about the relationship between AWMC and mild to moderate hearing loss in adults by comparing AWMC with respect to type, laterality and duration of hearing loss. This paper also examines current literature on auditory working memory (AWM) and provides a brief overview about working memory associated with various communication disorders in adults like hearing loss, attention-deficit/hyperactive disorder (ADHD), auditory processing disorders (APD) and learning disabilities (LD). Total 40 participants were involved in this study among that 20 were having hearing sensitivity within normal limits (control group) and other 20 were having mild to moderate hearing loss (experimental group). The results show that the AWMC of individuals with mild to moderate hearing loss is poor when compared to individuals with normal hearing. The poor AWMC in conductive and mixed hearing loss group when compared to sensor neural hearing loss group is also a major finding of the study. The paper concludes by highlighting the importance of assessing AWM in audiology clinical setting.
\end{abstract}

Keywords: Auditory working memory; Auditory working memory capacity; Mild to moderate hearing loss; Conductive hearing loss; Mixed hearing loss; Sensorineural hearing loss; Digit-span task

Abbreviations: AWM: Auditory Working Memory; AWMC: Auditory Working Memory Capacity; AWM: Adults with Normal/Mild-Moderate Hearing loss; DSB: Digit Span Backward; DSF: Digit span forward; LDSB: Longest Digit Span Backward; LDSF: Longest Digit Span Forward; MCL: Most Comfortable Loudness Level; PTA: Pure Tone Average; SDS: Speech Discrimination Scores; SRT: Speech Recognition Threshold; STM: Short Term Memory; WM: Working Memory; WMC: Working Memory Capacity; dB HL: Decibel Hearing Level

\section{Introduction}

Auditory working memory (AWM) is the process of keeping sounds in mind for short periods of time when the sounds are no longer present in the environment. The auditory feedback loop is the process of self-monitoring and correcting one's own speech. Auditory feedback is very important for the attainment of auditory skills and to acquire and produce fluent speech. Hearing is simply the act of perceiving sound by the ear. Hearing is a passive bodily process that occurs at subconscious level for a person with normal hearing. Listening, however, is something that we consciously choose to do. Hearing is essentially a passive bottom up driven process, whereas listening is a top down process that requires attention, many repetitions of stimuli, and tremendous cognitive coordination and effort. Hearing is a sense and listening is a learned skill.
To process sound effectively, the brain must receive maximal auditory information, to simultaneously receive, store, and process acoustic information, this phenomenon also referred to as working memory (WM). Short-term memory (STM) may be reflected or demonstrated through a person's ability to memorize a few or many items. WM involves more of cognitive processing of the same information.

WM refers to a brain system that provides temporary storage and manipulation of the necessary information for complex cognitive tasks such as language comprehension, learning, and reasoning. WM requires simultaneous storage and processing of information Baddeley [1]. Rudner and Lunner [2] define working memory capacity (WMC) as the cognitive ability that allows one to keep information within easy cerebral reach while 
simultaneously processing it. WM is so important that it may be the basis for general intelligence and reasoning, according to several studies (Dingfelder [3]; Stuart [4]; Gray and Bill, [5]).

The WM, serves as an interface between perception, longterm memory, and action (Baddeley, 2003). Despite, the physical absence of the sensory input, a representation of the information can be maintained and manipulated over a period of time (Baddeley, 2012). It is a defining feature that the cognitive resources constituting WM are limited with regard to the load of information that can be maintained i.e., memory load, as well as to the duration of how long information can be maintained i.e., memory decay. These constraints are inherently linked to the limited amount of attention that can be allocated to the tobe-remembered information (Gazzaley and Nobre, 2012). When limitations are exceeded, performance declines due to a lack of attention resources (Norman and Bobrow [6]).

The memory load refers to the number of items to be held in WM. Miller (1956) proposed that WMC in humans is approximately seven units of data, plus or minus two, and described this as the magical number seven. He claimed that the information-processing capacity of young adults is around seven elements which he called «chunks», regardless whether the elements are digits, letters, words, or other units. Later, this number has been revised to four chunks by Cowan (2001). In 2005, Cowan explains that to make precise predictions about how well working memory operate, one have to measure storage-specific capacity by preventing or controlling processing strategies like rehearsal and grouping and that is how one can observe a capacity limit of 3 to 5 separate items. In the case of auditory working memory, the acoustic degradation i.e., environmental noise, competing talkers or auditory degradation i.e., the lack of precision in neural encoding that accompanies sensorineural hearing loss (Bernstein and Oxenham, 2006; Humes and Roberts, 1990) will lead to increase memory load and decrease memory performance (Pichora-Fuller et al., 1995; Rudner et al., 2011).

Baddeley and Hitch [7] introduced the multi-component model of WM by revising the picture of STM provided by the Multi-Store Model (Atkinson and Shiffrin [8]). According to these authors, the components of working memory reflect their function and the type of information they process and manipulate. This study proposes that every component of WM has a limited capacity.

The components are,

(a) The central executive: The central executive is the most important component of the model. It is responsible for monitoring and coordinating the function of the slave systems and relates them to long term memory. It consists of two sub systems, the phonological loop and the visuospatial sketch pad.

(b) The phonological loop: The phonological loop deals with the spoken and written material. It consists of two parts; the phonological store (that is linked to speech perception information in speech based form for 1-2 seconds) and the articulatory control process (that is linked to articulation used to rehearse and store verbal information from the phonological store).

(c) The Visuospatial sketch pad: The visuospatial sketch pad deals with visual and spatial information.

(d) The episodic buffer: In 2000, Baddeley added an additional component called the episodic buffer. It links information across the long-term memory and the other components of WM.

It is relevant here to discuss about the difference between STM and WM as both are terms that have been frequently mentioned in the above paragraphs. The central executive region, located in the prefrontal cortex, seems to play a fundamental role in both STM and WM. Short term memory refers to a cognitive system that is used for holding sensory events, movements, and cognitive information, such as digits, words, names, or other items for a brief period of time (Kolb and Wishaw, 2009). The term WM became popular through the WM model of Baddeley and Hitch [7]. According to Baddeley [1], WM is defined as the maintenance and controlled manipulation of a limited amount of information before recall. Many studies support the concurrence of both STM and WM (Gathercole and Alloway [9]; Nadel and Hardt, [10]) but it is also claimed that the term WM has replaced the older term STM (Gray [11])

AWM is the process of actively maintaining sounds in memory over short periods of time (Kumar et al [12]). Baddeley [13] suggests that auditory short-term memory extends up to 5 or 10 seconds. The functioning of WM via the central executive system is suggested to be strongly dependent on the frontal lobes (Baddeley, 1996). Studies on nonhuman primates (GoldmanRakic [14]; Fuster,1989; Funahashi and Kubota [15]) and human subjects (Braver et al.,1997; Carlson et al., 1998; Martinkauppi et al., 2000) also indicate the importance of the prefrontal cortex in WM processing. The functional magnetic resonance imaging study in human by Kumar et al. (2016), demonstrates maintenance of single tones in memory is associated with activation in auditory cortex. In addition, sustained activation was observed in hippocampus and inferior frontal gyrus. Multivoxel pattern analysis showed that patterns of activity in auditory cortex and left inferior frontal gyrus distinguished the tone that was maintained in memory. Functional connectivity during maintenance was demonstrated between auditory cortex and both the hippocampus and inferior frontal cortex. The AWM is based on the maintenance of sound-specific representations in auditory cortex by projections from higher-order areas, including the hippocampus and frontal cortex (Kumar et al [12]).

WM appears to predict performance on a wide variety of tasks including reading comprehension (Daneman \& Carpenter [16]), phonological processing (Classon, Rudner, \& Ronnberg [17]), and attention (Kane \& Engle, 2003). WM also plays a key 
role in understanding speech, because listeners must decode the incoming speech signal while relating the information to stored knowledge and anticipating the speech that is forthcoming (Pichora-Fuller et al., 1995; Wingfield and Stine-Morrow, 2000; Akeroyd [18]). While listening to conversations, individuals must continually store and update auditory information that is spoken in real time. Therefore, a preserved WM is considered to be crucial for understanding speech and language (Caplan \& Waters, 1999; Daneman \& Carpenter [16]). Individual differences in WM are often observed (Brebion [19]) and are thought to be responsible for variability often seen in aging research (PichoraFuller \& Singh [20]; Unsworth [21]).

Craik [21] proposed that WM would appear to be an important factor in persons with hearing loss relative to speech understanding. When the audibility of the speech signal is reduced due to hearing loss, then more WM resources may need to be assigned when listeners are trying to comprehend the impoverished incoming speech signal (Foo et al., 2007; Rudner et al, 2011; Besser et al., 2013). Whenever listening becomes challenging, particularly in noisy environments, resources typically allocated to storage are actually used to process the difficult speech signal, limiting an individual's WM storage ability.

Degree of hearing loss refers to the severity of the loss. The degree of hearing loss commonly defined on the basis of the audiogram. The degree of hearing loss is usually described by the pure tone average (PTA), which is the mean of thresholds at speech frequencies, which are $500 \mathrm{~Hz}, 1000 \mathrm{~Hz}$ and $2000 \mathrm{~Hz}$. Clark (1981) classifies the degree of hearing loss as: -10 to 15 $\mathrm{dB}$ HL as Normal hearing; 16 to $25 \mathrm{~dB}$ HL as Slight hearing loss; 26 to $40 \mathrm{~dB} \mathrm{HL}$ as Mild hearing loss; 41 to $55 \mathrm{~dB} \mathrm{HL}$ as Moderate hearing loss; 56 to $70 \mathrm{~dB}$ HL as Moderately severe hearing loss; 71 to $90 \mathrm{~dB}$ HL as Severe hearing loss; $91+\mathrm{dB}$ HL as Profound hearing loss.

In case of mild hearing loss, people shows difficulty with hearing faint sounds or distant speech even in quiet situations (Stach, 2010). People with mild hearing loss have difficulty hearing soft consonant sounds making words seem incomplete. Noisy environments make hearing more challenging. They miss fragments leading to misunderstanding. The degree of difficulty experienced will depend upon the background noise level, distance from the speaker, and configuration of the hearing loss. The hearing loss at $30 \mathrm{~dB}$ can miss $25-40 \%$ of the speech signal; at $40 \mathrm{~dB}$ may miss 50\%, especially when voices are faint or speaker is not in line of vision. There will be high chances to miss unstressed words and consonants, especially when a high frequency hearing loss is present (Anderson and Matkin [22]).

People who suffer with moderate hearing loss will be able to engage in conversational speech only at close distance (Stach, 2010). Hard and soft consonant sounds become inaudible and speech becomes difficult to understand particularly with background noise. They understand conversational speech at a distance of 3-5 feet, if sentence structure and vocabulary are controlled. The amount of speech signal missed can be $50+\%$ with $40 \mathrm{~dB}$ loss and 80+\% with $50 \mathrm{~dB}$ loss (Anderson and Matkin [22]).

The extent to which the hearing problems cause a communication disorder depends on a number of auditory factors, including: degree of sensitivity loss, audiometric configuration, type of hearing loss, and degree and nature of a speech perception deficit. Confounding this issue further are individual patient factors like age of onset of loss, whether the loss was sudden or gradual, and communication demands on the patient, that are interrelated to these auditory factors (Stach, 2010).

It has been suggested that a lack of auditory input from an untreated hearing loss could negatively affect the neural networks involved in certain cognitive abilities (Sekuler and Blake (1987); Belin et al. [23]; Wong et al. (2010)). It has also been suggested that even mild hearing loss could lead to a decline in cognitive performance because the cognitive resources normally used for higher-level comprehension, like storing auditory information into memory, must be used by the individual to accurately decode and perceive the speech signal (Tun et al.(2009; Gosselin and Gagne (2011); Desjardins and Doherty $(2013,2014)$. Lin et al. [24], tracked the overall cognitive abilities including, concentration, memory and planning skills of 1984 adults whose average age was 77. After six years, those who began the study with hearing loss (severe enough to interfere with conversation), were 24 percent more likely than those with normal hearing to see their cognitive abilities diminish. The researchers suggest that, hearing loss seemed to speed up age related cognitive decline. The people with mild, moderate and severe degrees of hearing loss were projected to be two to five times more likely to develop dementia (Lin et al. [15]). A few studies have suggested that WM could be a predictor of overall success with amplification (Gatehouse, Naylor, and Elberling [25], Lunner and Sundewall-Thoren [26]). Ronnberg, Rudner, Lunner, and Zekveld [27] suggests that, the persons with higher WM scores perform better with faster signal processing speeds than persons with lower WM scores. However, another study by Cox and Xu (2010) reported that WM might be a more important factor for persons with lower WM than persons with high WM.

Amblyaudia is a new diagnostic category within Auditory Processing Disorders (APD). When one ear is developmentally deprived it causes the other ear to compensate and leads to weaknesses in the listener's binaural processing of auditory information (Moncrieff, 2012; Popescu and Polley [27]). People who experience temporary hearing loss, most commonly from ear infections, are at an increased risk of developing amblyaudia (Moncrieff, Demarest, Mormer and Littlepage, [28]). Characteristics of amblyaudia include speech comprehension difficulties, reading difficulties in children, information processing deficits, poor verbal working memory, poor adaptive skills, and inattention studies point to asymmetrical auditory 


\section{Global Journal of Otolaryngology}

input as the cause (Musiek and Weihing, 2011). Asymmetrical auditory input during periods of auditory development in children, leads to disruption in normal auditory development and results in neural impairment, which affects acoustic processing. Hearing loss is the primary disruption that leads to asymmetrical auditory input. The hearing loss associated with amblyaudia is conductive hearing loss. This is often due to fluid accumulation within the typically air-filled middle ear (RaeLynn, Lamminen and Houlihan, [29]).

Silman et al. (1984) demonstrated that an auditory deprivation effect may occur in adults when one ear regularly receives auditory input but the other ear is deprived of adequate auditory stimulation for a period of time. It is observed as a reduction in the speech recognition ability of the under stimulated ear. The auditory deprivation effect occurs in the unaided ear of patients with a bilateral sensorineural hearing loss who use monaural amplification. Their aided ears remain unchanged, as do both ears of similar patients using binaural amplification. This unaided ear effect has been repeatedly corroborated (Gelfand et al., 1987; Gatehouse, 1989, 1992; Stubblefield and Nye, 1989; Emmer, 1990; Silverman, 1989; Silverman and Silman 1990; Silman et al., 1992, 1993; Hurley, 1993; Poole and Jerger, 1994). Auditory deprivation effects also occur for the poorer ears of patients with asymmetric sensorineural hearing loss (Silverman and Emmer, 1993), and an analogous phenomenon effect has been reported in patients with Meniere's disease (Hood [30]). The auditory deprivation effect is not limited to adults. Gelfand and Silman (1993) states that children with bilateral moderate sensorineural hearing loss developed an auditory deprivation effect when using monaural hearing aids. Supportive findings were reported by Hattori (1993) for children with severe bilateral losses. The precipitating factors for the unaided ear effect are an unaided ear PTA1 >- $46 \mathrm{~dB}$ and a PTA2 >- $58 \mathrm{~dB}$, leading to the conclusion that the amount of hearing loss plays a major role in the onset of the unaided ear effect through protracted auditory deprivation (Hurley, [31]). According to Lin et al. (2013), adults with untreated hearing loss tend to develop a significant impairment in their cognitive abilities 3.2 years sooner than those with normal hearing. Those with a hearing impairment also experience a 30 to 40 percent greater decline in thinking abilities when compared to their counterparts without hearing loss (Lin, et al. 2013).

Literature shows the affected WM associated with several speech, language and communication disorders in adults. Few such disorders like attention-deficit/hyperactive disorder (ADHD), auditory processing disorders (APD) and learning disabilities (LD) are briefly discussed in the following paragraphs.

ADHD is a developmental neurobehavioral disorder characterized by a variety of persistent behavioral symptoms including inattention, hyperactivity, and impulsivity (American Psychiatric Association, 2000). Adler, Barkley, and Newcorn (2008) proposed that the hyperactivity and behavioral impassivity seen in children with ADHD may manifest as mental restlessness and excessive talking in adults. Adults with ADHD may also demonstrate difficulties with sustaining attention and organization (Wilens, 2007). Specific neurocognitive deficits identified in children with ADHD have included frontal lobe dysfunction characterized by executive function deficits (Sergeant, Geurts, and Oosterlaan, 2002), as well as difficulties with visuospatial working memory (Westerberg, Hirvikoski, Forssberg, and Klingberg, 2004). Similar neurocognitive deficits have also been observed in adults with ADHD. Boonstra, Oosterlaan, Sergeant, and Buitelaar's (2005) meta-analytic review identified deficits in executive functioning areas such as inhibition and set shifting, as well as in non-executive functioning areas such as fast word reading and color naming in adults with ADHD. Neurocognitive deficits in numerous other areas such as verbal and visual memory, and divided attention can also be maintained by adult ADHD patients even while on medication (Schoelin and Engel, 2005). In fact, memory deficits have frequently been identified as areas of difficulty in adults with ADHD and can include deficits in verbal WM (Lacene, 2004; Marchetta et al., 2008), spatial WM (McLean et al., 2004), and long- and short-term visual memory (Dige and Wik, 2005; Schoelin and Engel, 2005). Schweitzer, Hanford and Medoff (2006), assessed differences in WM functioning between normal adults and patients with ADHD (combined type and inattentive type), using sub-tests from the Wechsler Adult Intelligence Scale-III and Wechsler Memory Scale-III and the Paced Auditory Serial Addition Task (PASAT). The results showed that, the ADHD groups displayed significant weaknesses in contrast to the normal group on WM tests requiring rapid processing and active stimulus manipulation. No overall ADHD group subtype differences emerged; however, differences between the ADHD groups and the normal group varied depending on the measure and the gender of the participants. Gender differences in performance were evident on some measures of $\mathrm{WM}$, regardless of group, with males performing better than females.

Jerger and Musiek (2000) define APD as a deficit in the processing of information in the auditory modality. Moore (2007) describes an APD as a listening problem with hearing that cannot be explained by tests of peripheral auditory function. American speech and hearing association (2005) reports that auditory processing disorders may lead to or be associated with difficulties in higher order language, learning, and communication functions. Although auditory processing disorders may coexist with other disorders (e.g., ADHD, language impairment, and learning disability), it is not the result of these other disorders. APD is diagnosed on the basis of performance on a battery of auditory tests, which may include electrophysiologic as well as behavioral procedures, administered under acoustically controlled conditions (Jirsa and Clontz, 1990; Kraus et al, 1993; Musiek and Chermak, 1994; Chermak and Musiek, 1997). Children in the APD group had consistently lower scores than typically developing subjects in lateralization and WM capacity measures (Moossavi, Mehrkian, 


\section{Global Journal of Otolaryngology}

Lotfi, Faghihzadeh and Sajedi, 2014). Atcherson, Nagaraj, Kennett and Levisee (2015), observed various changes in memory and attention in older adults associated with APD. One of the universal findings in cognitive aging research is reduced speed of information (sensory and mental) processing with advancing age (Salthouse, 1996). This reduction in the speed of information processing along with reduction in cognitive skills is known to effect listening comprehension (Willott 1991, PickoraFuller 2003). Speech understanding especially in adverse or challenging environments has been related to listener's WM capacity (Akeroyd [18]; Holt and Lotto, 2008; Tun, Benichov and Wingfield, 2010). Shahidipour, Geshani, Jafari, Jalaie and Khosravifard (2013), investigated auditory memory in 47 elderly people, aged 60 to 80 by administering Dichotic Auditory Memory Test (DVMT). The subjects were divided in two groups, the first group consisted of elderly people with hearing range of 24 normal and the second one consisted of 23 elderly people with bilateral symmetrical ranged from mild to moderate sensorineural hearing loss in the high frequency due to aging in both genders. The results showed, significant difference was observed in DVMT between elderly people with normal hearing and those with hearing loss. According to the correlation test between PTA and the mean score of DVMT, increasing PTA resulted in reduction of DVMT score and this result was seen in both genders and all of the studied subjects. They suggested that, apart from aging, age-related hearing loss has shown significant effect on auditory verbal memory.

The term LD refers to a wide group of neurological disorders caused by deficits in the central nervous system which influence the individual's ability to maintain process or convey information to others in an efficient way (Kavale and Forness, 2000). The fifth edition of the Diagnostic and Statistical Manual of Mental Disorders introduced a new term, specific learning disorder, as a single diagnosis to describe all of these conditions (American Psychiatric Association, 2013). The most common LD is dyslexia, which affects a complex range of abilities related to reading and language, and studies shows that, $80 \%$ of people with LDs are dyslexic (Shaywitz 1998, 2003; Berninger and May, 2011). Other common types of LDs include dysgraphia, which affects writing abilities, dyscalculia, which affects the application of mathematical operations, and visual-spatial organization problems (Berninger and May, 2011). Nonverbal LDs are another type of learning difficulty whereby individuals demonstrate adequate verbal expression, vocabulary or reading skills, but have difficulties with certain nonverbal activities, such as problem-solving, visual-spatial tasks and reading body language or recognizing social cues (Handler and Fierson, 2011). Developmental dyslexia is associated with deficient memory skills and specific deficits in AWM have been reported (Swanson and Siegel, 2001; Torgesen, 1985). In adult readers with developmental dyslexia, deficient AWM skills may contribute to their reading and phonological deficits (Berninger et al., 2006). It has been suggested that impaired AWM skills may be one feature of LD (Banai and Ahissar, 2006; Berninger et al., 2006; Kibby et al., 2004). Swanson's (1994), comparison study on STM and WM in 75 children and adults with learning disabilities and 86 normally achieving children and adults shows that, for both ability groups, the factor analysis indicated that STM and WM loaded on different factors, and the regressions and partial correlations showed that these different factors accounted for separate variance in reading comprehension and mathematics. Both STM and WM are important in understanding reading comprehension and mathematics performance in children and adults with learning disabilities; however, WM is more important for children and adults without learning disabilities.

A research needs to be connected with work already done to attain an overall relevance and purpose. The studies related to AWM have stimulated enormous research activity in recent years throughout the world. The following paragraphs contain a summary of literature related to AWM in various combinations.

Millman and Mattys [32], conducted a study to assess the relationship between speech perception in modulated maskers and components of auditory verbal working memory (AVWM) over a range of signal to noise rations. They measured speech in noise and AVWM in 30 listeners (age range 31-67years) with normal hearing. AVWM was estimated using forward digit recall, backward digit recall, and non-word repetition. Their results show that speech perception in modulated maskers was related to individual differences in the phonological component of WM (as assessed by non-word repetition) but only in the least favorable signal to noise ration. The executive component of WM (as assessed by backward digit) was not predictive of speech perception in any condition. They concluded that, the listeners with greater phonological WMC are better able to correctly identify sentences in modulated noise backgrounds.

Barrett and Pilling [33], investigated the potential of measures of auditory short-term memory (ASTM) to provide a clinical measure of intrusion in tinnitus. They studied the response function for 6 normal listeners on a delayed pitch discrimination task were contrasted in three conditions designed to manipulate attention in the presence and absence of simulated tinnitus: (1) no tinnitus, (2) ignore tinnitus, and (3) attend tinnitus. The result of their study highlight that the delayed pitch discrimination functions were more variable in the presence of simulated tinnitus when listeners were asked to divide attention between the primary task and the amplitude of the tinnitus tone. This study indicates that the changes in the variability of ASTM may provide a novel means of quantifying the level of intrusion associated with the tinnitus percept during listening.

A comparison study of auditory and visual working memory functioning in college students with ADHD, LD, and clinical controls were done by Liebel and Nelson [34]. Their study was focused to investigate the role of ADHD subtype in WM functioning. The sample included 268 post-secondary students, 
which was divided into 4 groups: 110 had an ADHD diagnosis only, 72 had a LD diagnosis only, 35 had comorbid ADHD and LD diagnoses, and 60 individuals without either of these disorders comprise a clinical group. The study found that, there was no difference between auditory and visual WM functioning in ADHD only. In the learning disability group, AWM functioning was significantly weaker compared with visual WM. According to their study the individuals with ADHD did not demonstrate significant WM differences compared with clinical controls, but the individuals with a learning disability demonstrated weaker AWM than individuals in either the ADHD or clinical control groups.

Miller et al. [35] investigated the relationship between WM and speech recognition in noise with different noise types as well as in the presence of visual cues among adults with hearing loss. They did the study on 76 adults with bilateral, mild to moderately severe sensorineural hearing loss (mean age: 69 years). They used a cross sectional design and took 2 measures of WM: a reading span measure, and Word Auditory Recognition and Recall Measure (Smith, Pichora-Fuller and Alexander, 2016) Speech recognition was measured with the Multi-Model Lexical Sentence Test for Adults (Krik et al., 2012) in steady state noise and 4-talker babble, with and without visual cues. The conducted the testing in the unaided conditions. The results showed that, the visual cues and pure tone average are the only significant predictors of the Multi-Model Lexical Sentence Test outcomes. Neither WM measure nor noise type showed a significant effect.

Fullgrabe and Rosen [36], studied the importance of WM in speech in noise processing for listeners with normal hearing thresholds. To assess, they surveyed published and unpublished studies in which the Reading Span test was administered in conjunction with a measure of speech in noise (SiN) identification, using sentence material routinely used in audiological and hearing research. Their study revealed that, for young listeners with audiometrically normal hearing, individual variation in WMC are estimated to account for, on average, less than $2 \%$ of the that individual variations in WMC are predictive of SiN identification independently of the age and hearing of the listener.

\section{Need of the study}

For cognitive healthy adults with hearing loss, audiologists are less concerned with their listening processing as they already have intact language and cognitive skills. Thus, audiologists generally provide adequate and appropriate hearing technologies to make sound more accessible for these individuals. Several studies suggests that WM plays a key role in understanding speech (Pichora-Fuller et al., 1995; Wingfield and Stine-Morrow, 2000; Akeroyd, 2008) and also to predict performance on tasks including phonological processing (Classon, Rudner, \& Ronnberg [37]), and attention (Kane \& Engle, 2003).Therefore, it is considerably significant for an Audiologist to learn and to know about AWM skills in adults and the range of difference in AWM skills in normal hearing and hearing impaired individuals. The current study will be a comparison of the AWM in adults with normal hearing and mild to moderate hearing loss. This study examines the auditory working memory capacity (AWMC) of normal hearing adults and adults with mild to moderate hearing loss. The study will be helpful to know the influence of factors like, type of hearing loss (conductive, mixed and sensorineural), laterality of hearing loss (unilateral and bilateral) and duration of hearing loss on the AWMC in adults.

\section{Materials and Methods}

\section{Research Objectives}

The current study is aimed at exploring the auditory working memory capacity (AWMC) in normal hearing adults and adults with mild to moderate hearing loss. In particular, the following areas of interests were examined:

i. Understand the difference in AWMC in normal hearing adults and adults with mild to moderate hearing loss.

ii. Understand the differences and similarities in AWMC with respect to mild to moderate conductive or mixed hearing loss and mild to moderate sensorineural hearing loss in adults.

iii. Understand the differences and similarities in AWMC with respect to unilateral and bilateral mild to moderate hearing loss in adults.

iv. Understand differences and similarities in AWMC with respect to duration of the hearing loss in adults.

\section{Subjects}

Forty participants between age group of 25-60 years served as subjects for this study. Out of 40, 20 (11 females and 9 males) were having hearing sensitivity within normal limits (control group), and other 20 (7 females and 13 males) were having mild to moderate hearing loss (experimental group). In the experimental group, 11 individuals were diagnosed with conductive and mixed hearing loss and 9 with sensorineural hearing loss. Bilateral hearing loss were observed in 8 and unilateral hearing loss in 12. Duration of hearing loss were grouped in to three, (1) duration of hearing loss less than 1 year, that was noted in 9 individuals, (2) duration of hearing loss 1 to 5 years were reported in 7 individuals and (3) duration of hearing loss greater than 5 years were noted in 4 individuals.

\section{Inclusion criteria}

1. 20 participants were adults with normal hearing with normal PTA, good speech discrimination scores and A type tympanogram.

2. 20 participants were adults with mild to moderate hearing loss, with fair to good speech discrimination scores.

3. All participants were well proficient in English language. 
4. All participants were adults between the age group of 25- 60 years.

\section{Exclusion criteria}

1. Adults with current complaint of tinnitus, vertigo and otalgia were excluded.

2. Adults with any neurogenic or psychogenic disorder were excluded.

3. Adults with poor speech discrimination scores.

4. Adults with minimal, moderately severe, severe and profound hearing loss.

5. Adults with a sudden onset of hearing loss.

6. Adults, who are using amplification.

\section{Procedure}

All the tests were done in a double walled acoustically treated room with a single walled control room. All the participants were seated comfortably and instructed well before the administration of each tests. An Equinox 2.0 Interacoustics clinical diagnostic audiometer and a Titan Interacoustics clinical diagnostic tympanometer, calibrated to meet current specification (ANSI S3.6-2010) was used to administer the tests. All the participants were undergoing the following 4 tests.

1. Tympanometry

2. Pure Tone Audiometry

3. Speech Audiometry

4. Forward and Backward digit span task

Case history: An important starting point of any audiological evaluation is case history. It provides necessary information about the nature of auditory complaints, including whether it is one ear or both, whether it is acute or chronic, and the duration of the problem (Stach, 1998). All the participants were undergoing a brief case history prior to the audiological evaluations, which was focused to gather information about duration of hearing problem, family history, history of noise exposure, history of ototoxicity, presence of tinnitus, nature of hearing loss, ears affected (unilateral/bilateral), history of amplification, history of dizziness and any other medical history.

Tympanometry: Tympanometry is a test of middle ear functioning. It records the compliance of the tympanic membrane to changing air pressures, indicating how effectively sound is transmitted into the middle ear. Tympanometry assists in the detection of fluid in the middle ear, perforation of the eardrum, or any other kind of middle ear abnormalities. The result is graphically represented, called tympanograms. A typical tympanometry result indicates the ear canal volume ( $\mathrm{cm} 3$ ), the maximum pressure ( $\mathrm{daPa}$ ) and the peak compliance $(\mathrm{ml})$. Impedance testing is crucial in distinguishing a conductive loss from a sensorineural hearing loss.
All the participants were seated comfortably and instructed to remain as still as possible (avoid any unnecessary movement and avoid speaking or swallowing after the probe has been inserted) during the test. Tympanograms were obtained using a $226-\mathrm{Hz}$ probe tone.

All the subjects in the control group got A-type tympanogram, which suggests normal middle ear function. The participants in the experimental group showed different types of tympanograms. Nine of them got A- type tympanogram, suggestive of normal middle ear function, which was accompanied with sensorineural hearing loss in the pure tone audiogram. C, Cs and B type tympanogram that suggests some kind of middle ear dysfunction were obtained for 11 participants. C and Cs-type was noted in 3 and 2 participants respectively and B-type was obtained for 6 participants, those were accompanied with conductive and mixed hearing loss in the pure tone audiogram.

Pure Tone Audiometry: Pure tone audiometry is the measurement of an individual's hearing sensitivity for calibrated pure tones. It determines the faintest tones an individual can hear at selected frequencies. Pure tone audiometry includes manual air-conduction (AC) measurements at 250, 500, 1000, $2000,3000,4000,6000$, and $8000 \mathrm{~Hz}$ plus bone-conduction (BC) measurements at octave intervals from $250 \mathrm{~Hz}$ to $4000 \mathrm{~Hz}$ and at $3000 \mathrm{~Hz}$ as needed. Also, when required appropriate masking is used.

The diagnostic audiometer used for the study was Equinox 2.0 Interacoustics, was calibrated to meet the specifications of ANSI S3.6-2010. The AC thresholds were estimated by using a supra-aural head phones (TDH-39) and BC thresholds with a bone vibrator (B-72).

Appropriate verbal instructions were given to all the participants prior to the $\mathrm{AC}, \mathrm{BC}$ and masking measurements. The participants were instructed to indicate the faintest tone that heard by pressing and releasing the signal switch.

The thresholds were obtained using a modified HughsonWestlake down-up procedure. When a difference of $20 \mathrm{~dB}$ or more exists between the threshold values at any two adjacent octave frequencies from 500 to $2000 \mathrm{~Hz}$, interoctave measurements were made. After the threshold estimation in all the frequencies, a pure-tone average was calculated in each ear by taking the average of hearing thresholds at 500,1000, and 2000, which was considered to determine the degree of hearing loss. The air-bone gap was also noted to determine the type of hearing loss.

Speech Audiometry: Speech audiometry refers to procedures that use speech stimuli to assess auditory function (Konkle and Rintelmann, 1983). The participants in this study underwent 3 speech audiometry tasks, speech recognition threshold (SRT), speech discrimination scores (SDS) and most comfortable loudness level (MCL). The speech stimuli were presented through a pair of supra-aural headphones (TDH-39) 
with calibrated (ANSI S3.6-2010) Equinox 2.0 Interacoustics diagnostic audiometer. All the speech stimuli were presented live via VU meter monitor, with voice intensity was balanced at $0 \mathrm{~dB}$ and without lip reading.

Speech recognition threshold is the minimum intensity at which the speech stimuli can be understood. The threshold was estimated as intensity in $\mathrm{dB}$ HL at which $50 \%$ of the words are properly repeated. All the participants were instructed to repeat the words as they heard. The stimuli used were Spondee words and the initial presentation level was PTA + 20dB HL. The descending method (Tillman, 1973) was used in this study to obtain the SRT.

Speech discrimination scores is a procedure of establishing the percentage of correctly perceived phonetically balanced monosyllabic words or consonant vowel combination presented at a comfortable supra-threshold level. This is to find out speech discrimination ability of an individual. Carhart (1965) recommended the use of monosyllabic words for discrimination test since they are meaningful to the patient and are nonredundant. Prior to the task, all the participants were instructed well to repeat the words as they heard. The stimuli used in the study was NU 6-word list (from Gelfand, 2001), which were presented at the level, SRT $+40 \mathrm{~dB}$ HL. The scores were calculated and represented in percentage. All the participants scored above $70 \%$, which can consider as fair to good speech discrimination skills.

Most comfortable loudness level is the hearing level at which, an individual experiences speech stimulus to be most comfortable or finding out the intensity level at which the patient prefers to listen to speech material. The speech stimuli used here was live cold running speech. The rainbow passage. The participants were instructed to rate the level at which listening is found to be most comfortable. Several trials were completed to obtain a specific level or a single value. The level obtained was noted as intensity in dB HL. Digit span task: A digit span task is used to measure WMC. Participants hear a sequence of numerical digits and are tasked to recall the sequence correctly, with increasingly longer sequences being tested in each trial. The participant's auditory memory span is the longest number of sequential digits that can accurately be remembered. Digit span tasks can be given forwards or backwards, once the sequence is presented, the participant is asked to either recall the sequence in normal or reverse order (Blankenship, 1938). Digit-span tasks are the most commonly used test for AWM span, partially because performance on a digit-span task cannot be affected by factors such as semantics, frequency of appearance in daily life, complexity, etc (Jones, Gary; Macken, Bill, [38]).

Verbal / auditory working memory is also thought to be one of the elements underlying intelligence thus, the digit span task is a common component of many IQ tests, including the widely used Wechsler Adult Intelligence Scale (WAIS) (Schroeder, et.al, 2011; Heinly et.al, 2005). In the present study the digit span task was taken from WAIS-IV. The tasks given for the participants were the Digit Span Forward (DSF) and Digit Span Backward (DSB).

Digit Span Forward (DSF): DSF consist of string of numbers from 2 digits to 9 digits. There are 8 items with each item consist of two trials. The examiner needs to administer both the trials even if the listener passes trail 1. The digit strings will increase in length with each trial.

In the present study the digits were presented through a pair of supra-aural headphones (TDH-39) with calibrated (ANSI S3.6-2010) Equinox 2.0 Interacoustics diagnostic audiometer. The mode of presentation was live via VU meter monitor, with voice intensity was balanced at $0 \mathrm{~dB}$.In case of unilateral hearing loss the stimuli were presented in the affected ear and stimuli were presented binaurally for bilateral hearing loss. The presentation level was at the level of participant's MCL. Each trial verbatim was read at the rate of one digit per second, in an even monotone without any variation in the pitch of voice. The task was discontinued after obtained scores of 0 on both trials of an item.

All the participants were seated comfortably in an acoustically treated room and instructed to listen carefully and repeat the numbers in the same sequence as they heard. Digit Span Backward (DSB): DSB consist of another string of numbers from 2 digits to 8 digits. In DSB the first item is a sample item and that won't be scored. The scoring will start from item 1 and like DSF, DSB also have 8 items with each item consist of two trials. The study followed the same procedures that mentioned above for DSF, except the instruction part. For DSB, all the participants were instructed to listen carefully and repeat the heard numbers backwards, or in the reverse order.

Scoring of digit span tasks: For each trial in DSF and DSB, a correct response was scored as 1 point and incorrect response or no response as 0 point. The item score is the sum of the scores on the two trials for that item. The DSF total raw score is obtained by summing the item scores on DSF. The maximum DSF total raw score will be 16 points. The DSB total raw score is obtained by summing the item scores on DSB (sample trials were not included). The maximum DSB total raw score will be 16 points. The longest digit span forward (LDSF) score were also obtained, which was the number recalled on the last DSF trial scored 1 point. The maximum LDSF score will be 9 points. Similarly, the longest digit span backwards (LDSB) was the number of digits recalled on the last DSB trial scored 1 point. The maximum LDSB score will be 8 points.

\section{Method of statistical analysis}

The data emerging from study was coded for computer analysis. Quantitative analysis was carried out using the Statistical Package for Social Sciences Version 15.0 for Windows (SPSS version 15.0). Quantitative analysis included parametric and non-parametric statistical tests. Descriptive statistics were used to analyze the data obtained at baseline and on the clinical and socio-demographic variables, including frequencies, mean 


\section{Global Journal of Otolaryngology}

and standard deviation. Difference between the groups was analyzed using independent sample t-test, since it compares the mean differences between two independent groups on a given variable. One-way analysis of variance (ANOVA), which compares the mean of three or more groups based on a independent variable, was used to determine the difference in DSF, LDSF, DSB, LDSB on variables such as type and duration of hearing loss in the experimental group. The difference between the groups on categorical variable gender was analyzed using Chi-Square test, since it determines the association between two categorical variables, and the quantitative variable age was analyzed using independent sample t-test. The significance level has been kept as 0.05 .

\section{Results}

The study was done to understand the nature of AWM in adults with normal hearing and adults with mild to moderate hearing loss. A sample of 40 adults was analyzed for the study. Of the 40, 20 were adults with normal hearing and other 20 were adults with mild to moderate hearing loss. The DSF and DSB tasks from WAIS- IV were used to measure the AWMC of all the participants. The data were analyzed across the two groups (adults with normal hearing and adults with mild to moderate hearing loss) and also across three categories in the hearing loss group (type of hearing loss, laterality of hearing loss, and duration of hearing loss).

The socio-demographic data such as age and gender of the experimental group and control group were analyzed using t-test and Chi-square test. The results are shown below in Table 1. The table indicates the mean value and standard deviation of age as 42.1 and 10.77 for the participants in experimental group and control group as 41 and 10.15. The t value shows 0.33 and the two-tailed significance shows 0.74 , which indicate there is no significant difference Table $5 \& 6$.

Table 1: Socio-demographic data of experimental and control group (Age using t-test, gender using Chi-Square test).

\begin{tabular}{|c|c|c|c|c|c|c|c|}
\hline \multicolumn{2}{|c|}{ Variable } & $\begin{array}{l}\text { Experimental Group } \\
\qquad(\mathrm{N}=20)\end{array}$ & & $\begin{array}{c}\text { Control Group } \\
(\mathrm{N}=20)\end{array}$ & & 't' value & Significance (2 tailed) \\
\hline \multirow{3}{*}{\multicolumn{2}{|c|}{ Age }} & Mean & $\pm *$ SD & Mean & $\pm \mathrm{SD}$ & & \\
\hline & & 42.1 & 10.77 & 41 & 10.15 & 0.33 & 0.74 \\
\hline & & ${ }^{*} \mathrm{~N}$ & & $\mathrm{~N}$ & & Chi Square Value & Significance (2 sided) \\
\hline \multirow[t]{2}{*}{ Gender } & Male & \multirow{2}{*}{\multicolumn{2}{|c|}{$\begin{array}{c}13 \\
7\end{array}$}} & \multirow{2}{*}{\multicolumn{2}{|c|}{$\begin{array}{c}9 \\
11\end{array}$}} & 1.62 & 0.34 \\
\hline & Female & & & & & & \\
\hline
\end{tabular}

Table 2: Description of clinical variables in experimental group.

\begin{tabular}{|c|c|c|}
\hline Variable & Experimenta Group (N= 20) & *N \\
\hline Laterality of & Unilateral & 12 \\
\hline hearing Loss & Bilateral & 9 \\
\hline $\begin{array}{c}\text { Duration of } \\
\text { hearing loss }\end{array}$ & Less than one year & 7 \\
\cline { 2 - 3 } & One to five years & 4 \\
\cline { 2 - 3 } $\begin{array}{c}\text { Type of hearing } \\
\text { loss }\end{array}$ & More than 5 years & 7 \\
\cline { 2 - 3 } & Conductive & 4 \\
\cline { 2 - 3 } & Mixed & 9 \\
\hline
\end{tabular}

*N- Frequency

Table 3: Comparison of the Experimental group and the Control Group on the DSF, LDSF, DSB, and LDSB. (t-test).

\begin{tabular}{|c|c|c|c|c|c|c|}
\hline \multirow{2}{*}{ Variable } & \multicolumn{2}{|c|}{ Experimental group $(\mathrm{N}=20)$} & \multicolumn{2}{|c|}{ Control group $(\mathrm{N}=\mathbf{2 0})$} & \multirow{2}{*}{ t value } & \multirow{2}{*}{$\begin{array}{l}\text { Significance } \\
\text { (Two tailed) }\end{array}$} \\
\hline & Mean & $\pm * \mathbf{S D}$ & Mean & \pm SD & & \\
\hline DSF & 6.75 & 1.16 & 9.15 & 1.42 & -5.83 & $\mathrm{p}<0.001$ \\
\hline LDSF & 4.85 & 0.67 & 6.75 & 0.91 & -7.51 & $\mathrm{p}<0.001$ \\
\hline DSB & 6.30 & 1.08 & 8.35 & 0.81 & -6.78 & $\mathrm{p}<0.001$ \\
\hline LDSB & 3.65 & 0.93 & 5.05 & 0.39 & -6.18 & $\mathrm{p}<0.001$ \\
\hline
\end{tabular}

*SD - Standard Deviation 


\section{Global Journal of Otolaryngology}

Table 4: Comparison of the study variables DSF, LDSF, DSB, and LDSB with respect to type of hearing loss. (One-way ANOVA).

\begin{tabular}{|c|c|c|c|c|c|c|c|c|}
\hline \multirow{2}{*}{ Variable } & \multicolumn{2}{|c|}{ Conductive $(N=7)$} & \multicolumn{2}{|c|}{ Mixed $(\mathrm{N}=4)$} & \multicolumn{2}{|c|}{ Sensorineural $(N=9)$} & \multirow{2}{*}{ f value } & \multirow{2}{*}{ Significance } \\
\hline & Mean & $\pm *$ SD & Mean & \pm SD & Mean & \pm SD & & \\
\hline DSF & 6.70 & 1.11 & 6.25 & 1.50 & 7.00 & 1.11 & 11.32 & $\mathrm{p}<0.001$ \\
\hline LDSF & 4.70 & 0.75 & 4.75 & 0.95 & 5.00 & 0.50 & 18.29 & $\mathrm{p}<0.001$ \\
\hline DSB & 6.00 & 0.57 & 5.50 & 0.57 & 6.88 & 1.26 & 20.39 & $\mathrm{p}<0.001$ \\
\hline LDSB & 3.40 & 0.78 & 3.00 & 0.00 & 4.11 & 1.05 & 18.17 & $\mathrm{p}<0.001$ \\
\hline
\end{tabular}

Table 5: Comparison of the study variables DSF, LDSF, DSB, and LDSB with respect to laterality of hearing loss (t-test).

\begin{tabular}{|c|c|c|c|c|c|c|}
\hline \multirow[t]{2}{*}{ Variable } & \multicolumn{2}{|c|}{ Unilateral hearing loss $(\mathrm{N}=12)$} & \multicolumn{2}{|c|}{$\begin{array}{l}\text { Bilateral hearing } \\
\text { Loss }(\mathrm{N}=8)\end{array}$} & \multirow[t]{2}{*}{ t value } & \multirow{2}{*}{$\begin{array}{l}\text { Significance } \\
\text { (Two tailed) }\end{array}$} \\
\hline & Mean & $\pm *$ SD & Mean & \pm SD & & \\
\hline DSF & 6.83 & 1.19 & 6.63 & 1.19 & 0.38 & 0.71 \\
\hline LDSF & 4.92 & 0.79 & 4.75 & 0.46 & 0.53 & 0.60 \\
\hline DSB & 6.25 & 1.06 & 6.38 & 1.19 & 0.25 & 0.81 \\
\hline LDSB & 3.58 & 1.19 & 3.75 & 1.04 & 0.38 & 0.71 \\
\hline
\end{tabular}

*SD - Standard Deviation

Table 6: Comparison of the study variables DSF, LDSF, DSB, and LDSB with respect to duration of hearing loss (One-way ANOVA).

\begin{tabular}{|c|c|c|c|c|c|c|c|c|}
\hline \multirow[t]{2}{*}{ Variable } & \multicolumn{2}{|c|}{ Less than one year $(\mathrm{N}=9)$} & \multicolumn{2}{|c|}{ One to five years $(\mathrm{N}=7)$} & \multicolumn{2}{|c|}{ More than 5 years $(\mathrm{N}=4)$} & \multirow[t]{2}{*}{ f value } & \multirow[t]{2}{*}{ Significance } \\
\hline & Mean & $\pm *$ SD & Mean & \pm SD & Mean & $\pm \mathrm{SD}$ & & \\
\hline DSF & 7.10 & 1.05 & 6.70 & 1.30 & 6.00 & 0.8 & 1.79 & 0.20 \\
\hline LDSF & 5.10 & 0.78 & 4.70 & 0.48 & 4.50 & 0.57 & 1.43 & 0.27 \\
\hline DSB & 6.40 & 1.23 & 6.10 & 0.89 & 6.25 & 1.25 & 0.50 & 0.62 \\
\hline LDSB & 3.60 & 1.00 & 3.70 & 0.90 & 3.50 & 1.00 & 0.52 & 0.61 \\
\hline
\end{tabular}

*SD - Standard Deviation

The table also shows the frequency of male and female gender across the two groups. In experimental group the frequency of male and female was 13 and 7 , and in the control group it was 9 , and 11 , respectively. The chi-square value was obtained as 1.62 . The two-sided significance shows 0.34 , that indicates no significant differences.

Table 2 shows the description of clinical variables in experimental group, such as laterality of hearing loss (unilateral and bilateral), duration of hearing loss (less than 1-year, 1- 5 years and more than 5 years) and type of hearing loss (conductive, mixed and sensorineural), in terms of frequency.

According to the above table the frequency of unilateral and bilateral hearing loss are 12 , and 8 respectively. The frequency of the duration of the hearing loss shows 9 in the less than 1-year group, 7 in 1-5 years group and 4, in more than 5 years group. The frequency of the conductive mixed and sensorineural hearing loss is represented as 7, 4, and 9 respectively Table 4 .

The Table 3 shows the comparison of experimental and control group with respect to the scores obtained for DSF, LDSF, DSB, and LDSB were done using independent sample t-test. The above table depicts the mean and standard deviation values of the scores obtained by the participants in experimental and control groups for DSF, LDSF, DSB and LDSB. Here the control group shows the higher mean and standard deviation values compared to experimental group. The $t$ values for the tasks DSF, LDSF, DSB, and LDSB are $-5.83,-7.51,-6.78$, and -6.18 respectively. The twotailed significance is obtained as $<0.001$ for all the four variables, which indicates that there is significant difference.

comparison of the study variables such as DSF, LDSF, DSB, and LDSB with respect to the type of hearing loss i.e., conductive, mixed and sensorineural hearing loss were done using one-way ANOVA. The f values obtained for the tasks were 11.32 for DSF, 18.32 for LDSF, 20.39 for DSB, and 18.17 for LDSB. The significant value obtained across all the study variables were less than 0.05 , indicates that there is significant difference in the study variables with respect to the type of hearing loss. The mean values show that the sensor neural hearing loss scored higher compared to conductive and mixed hearing loss, whereas the conductive and mixed hearing loss scored almost same across all the tasks.

comparison of the study variables such as DSF, LDSF DSB, and LDSB with respect to the laterality of the hearing loss i.e., unilateral and bilateral hearing losses were obtained by using independent sample t-test. The mean and standard deviation values obtained by unilateral and bilateral hearing loss groups, shows almost the same across all the four tasks (DSF, LDSF, DSB, and LDSB). The t values for the tasks DSF, LDSF, DSB, and LDSB were obtained as $0.38,0.53,0.25$ and 0.38 respectively. The two- 


\section{Global Journal of Otolaryngology}

tailed significant value for the DSF is 0.71 , LDSF is 0.6 , DSB is 0.81 and LDSF is 0.71 , which indicates no significant differences.

One-way ANOVA was used to find out the co relation of the study variables DSF, LDSF, DSB and LDSB with respect to the duration of the hearing loss i.e., less than 1 year, 1-5 years, and more than 5 years. The above Table 6 shows the mean and standard deviation of the three groups across the tasks DSF, LDSF, DSB, and LDSB. The f values for the variables DSF, LDSF, DSB, and LDSB were obtained as $1.79,1.43,0.50$ and 0.52 respectively. The significant values for all the tasks were found greater than the significant level, suggests that no significant differences.

\section{Discussion}

The first objective of the study was to understand the difference in AWMC in normal hearing adults and adults with mild to moderate hearing loss. The results in the Table 3 shows that, there are significant differences in the level of auditory working memory in terms of DSF, LDSF, DSB and LDSB among the adults with normal hearing and adults with mild to moderate hearing loss. The mean values indicate that the adults with normal hearing showed higher scores across all the 4 variables compared to adults with mild to moderate hearing loss. This finding suggests that the hearing / hearing loss (mild to moderate) is a factor that can influence the AWMC of an individual. Lin et al. [24], also explained the experience of 30 to 40 percent greater decline in cognitive abilities with hearing impairment when compared to their counterparts without hearing loss. Several previous studies by Tun et al. (2009); Gosselin and Gagne (2011); Desjardins and Doherty (2013, 2014) suggests that mild to moderate hearing loss could lead to a decline in cognitive performance. These findings support the current study results indicating poor AWM (which is a cognitive system (Baddeley $[39,40]$ ), in adults with mild to moderate hearing loss by comparison with normal hearing adults.

The second objective of the study was to understand the relationship of AWMC with type of hearing loss (conductive, mixed and sensorineural hearing loss). The results show that there is a significant deference in auditory working memory capacity in between the 3 groups, the conductive, mixed and sensorineural hearing loss (Table 4). The mean values show that the conductive and mixed hearing loss group performed poorer compared to sensorineural hearing loss group, which shows that, conductive and mixed hearing loss in adults is also a prominent factor that can influence the AWMC along with sensorineural hearing loss. Currently, there is no information regarding the co relation between conductive/mixed hearing loss and AWMC in adults. But several previous studies indicate the auditory temporal processing deficits associated with conductive hearing loss. Auditory temporal processing determines understanding of speech, appreciation of music, being able to localize a sound source, and to listen to a person in a noisy crowd (Eggermont, 2015). Xu et al [41] revealed that conductive hearing loss significantly alters temporally-precise properties of auditory cortex synapses and spikes, and this may contribute to auditory temporal processing deficits caused by mild to moderate hearing loss. Bayat et.al (2017) also reported reduced auditory temporal processing ability in adults with conductive hearing loss compared to normal hearing subjects.

The third and fourth objective of the study was to compare the AWMC of adults in terms of laterality and duration of the hearing loss. The study shows that there was no significant difference in the AWMC with respect to unilateral and bilateral hearing loss (Table 5). The AWMC comparison with respect to duration of hearing loss that shows in Table 6 also indicates no significant differences. This concludes that, according to the current study the laterality of hearing loss and the duration of hearing loss are not the major factors that can influence the AWMC in adults with mild to moderate hearing loss [42-47].

There are limitations of the current study that warrant further research. First, the relatively small sample size in the experimental group limited the power of the current analysis to detect relationships among variables like laterality of hearing loss and duration of hearing loss, while its likely that the additional participants may have strengthen the findings. Secondly this study couldn't do the alternative cognitive assessment to consider the other variables that can influence the AWMC, such as cognition, mental status, academic background, etc. Also, the stimulus used in study to measure the AWMC was only with one kind [48-53] stimulus i.e., Forward and backward digit span tasks, which consist of only numbers. Finally, even though the age range in the current study covers young adults to older adults, the study couldn't group participants according to the age to rule out the age factor that can influence the results [54-75].

Despite these limitations, the results of the current study support a growing body of literature indicating that the AWMC, which is an important cognitive function that is crucial for effective verbal communication (Caplan \& Waters, 1999; Daneman \& Carpenter [16]), is poor in adults with mild to moderate hearing loss when compared to normal hearing adults. The study gains the attention towards the co relation of AWMC and the type of hearing loss, which concludes that conductive and mixed hearing loss can also lead to poor AWMC in adults with mild to moderate hearing loss [76-85] Appendix.

\section{Conclusion}

Hearing impairment is a prevalent and a universal health concern that have a great impact on a person's quality of life, both physically and emotionally. Several studies suggest that WM is important in individuals with hearing loss relative to speech understanding (Craik (2007; Foo et al., 2007; Rudner et al, 2011; Besser et al., 2013). The present study results indicate the AWMC of individuals with mild to moderate hearing loss is poor when compared to individuals with normal hearing. It may contribute 
the hearing-impaired listeners likely to have greater difficulty in processing complex or lengthy discourse than their normal hearing peers (Foo et al., 2007; Rudner et al, 2011; Besser et al., 2013). The poorer AWMC in conductive and mixed hearing loss group when compared to sensorineural hearing loss group, also gains the attention that the individuals with conductive/ mixed hearing loss also may get difficulty in comprehending speech. Ferfuson and Henshaw (2015), proposes that an integrated auditory-cognitive training approach may increase the benefit of training for people with hearing loss in terms to improve the speech understanding in adverse conditions. Few studies have suggested that WM could be a predictor of overall success with amplification (Gatehouse, Naylor, and Elberling, 2003; Lunner and Sundewall-Thoren, 2007).

If $\mathrm{AWMC}$ has the potential to influence speech processing ability and apparently the amplification, then it seems admissible that the Audiologists may seek ways to assess AWM in the clinical setting. Future studies should use more consistent materials and methodological approaches to illuminate a better understanding regarding the possible associations between AWM and hearing loss in more ecologically relevant conditions.

\section{References}

1. Baddeley A (1992) Working Memory. Science 255(5044): 556-9.

2. Ng EHN, Rudner M, Lunner T, Pedersen MS, Rönnberg J (2013) Effects of noise and working memory capacity on memory processing of speech for hearing-aid users. International Journal of Audiology, 52(7): 433-441.

3. Dingfelder SF (2005) A Workout for Working Memory. In the American Psychological Association's Monitor on Psychology. Cover Story 36(8): 48.

4. Stuart A (2014) What Is Working Memory and Why Does it Matter? National Center for Learning Disabilities.

5. Gray J, Bill M (2015) Questioning Short Term Memory amd its Management: Why Digit Span Measures Long term associate learning. Cognition 144: 1-13.

6. Baddeley AD, Thomson N, Buchanan M (1975) Word length and the structure of short-term memory. Journal of Verbal Learning and Verbal Behavior 14: 575-589.

7. Baddeley AD, Hitch G (1974) Working memory. Psychology of Learning and Motivation 8: 47-89.

8. Atkinson RC, Shiffrin RM (1968) Human memory: a proposed system and its control processes. In: Spence KW, Spence JT, editors. The Psychology of Learning and Motivation: Advances in Research and Theory New York, USA.

9. Gathercole SE, Alloway TP (2006) Practitioner review: short-term and working memory impairments in neurodevelopmental disorders: diagnosis and remedial support. Journal of Child Psychology and Psychiatry 47(1): 4-15.

10. Nadel L, Hardt $O$ (2011) Update on memory systems and processes. Neuro psychopharmacology 36: 251-273.

11. Gray P (2007) Memory and consciousness in Psychology, $5^{\text {th }}$ Edn, (In: Eds: Gray P New York: Worth Publishers).

12. S Kumar, S Joseph, PE Gander, N Barascud, AR Halpern, et al. (2016) A Brain System for Auditory Working Memory. Journal of Neuroscience 36(16): 4492-4505.
13. Baltes PB, Lindenberger U (1997) Emergence of a powerful connection between sensory and cognitive functions across the adult life span: a new window to the study of cognitive aging? Psychol Aging 12(1): 1221.

14. Stach B, Loiselle L, Jerger J, Mintz S, Taylor C (1987) Clinical experience with personal FM assistive listening devices. The Hearing Journal 40: 24-30.

15. Lindenberger U, Baltes PB (1994a) Aging and intelligenc. In Eds: The Encyclopedia of Human Intelligence, eds Sternberg RJ, Ceci SJ, Horn JL, Hunt E, Matarazzo JD, Scarr S, editors (pp. 52-66): Macmillan ,New York, USA.

16. Daneman M, Carpenter PA (1980) Individual differences in working memory and reading. Journal of Verbal Learning and Verbal Behavior 19(4): 450-466.

17. Rudner M, Lunner T (2013) Cognitive Spare Capacity as a Window on Hearing Aid Benefit. Seminars In Hearing 34: 298-307.

18. Akeroyd MA (2008) Are individual differences in speech reception related to individual differences in cognitive ability? A survey of twenty experimental studies with normal and hearing-impaired adults. Int J Audio 47(Suppl 2): S53-S71.

19. Berlin CI, Weyand TG (2003) The Brain and Sensory Plasticity: Language Acquisition and Hearing. Clifton Park, NY: Thompson Delmar Learning.

20. Pichora-Fuller MK (2008) Use of supportive context by younger and older adult listeners: balancing bottom-up and top-down information processing. International Journal of Audiology 47: S72-S82.

21. Craik FIM (2007) Commentary - The role of cognition in age-related hearing loss. Journal of American Academy of Audiology 18: 539-547.

22. Anderson K, Matkin N, (2007) Relationship of Hearing Loss to Listening and Learning Needs, revision and updating of 1991 and 1998 versions.

23. Belin P, Zatorre RJ, Hoge R, Evans AC, Pike B (1999) Event-related fMRI of the auditory cortex. Neuroimage 10(4): 417-429.

24. Lin FR, Yaffe K, Xia J, Xue QL, Harris TB, Purchase-Helzner E, et al. (2013) Hearing loss and cognitive decline in older adults. JAMA International Medicine 173(4): 293-299.

25. Gatehouse S, Naylor G, Elberling C (2003) Benefits from hearing aids in relation to the interaction between the user and the environment. International Journal of Audiology 42 (Suppl 1) S77-S85.

26. Lunner Y, Rudner M, Ronnberg J (2009) Cognition and hearing aids. Scandinavian Journal of Psychology 50(5): 395-403.

27. Rönnberg J, Rudner M, Lunner T, Zekveld AA (2010) When cognition kicks in: working memory and speech understanding in noise. Noise Health 12(49): 263-269.

28. Popescu MV, Polley DB (2010) Monaural Deprivation Disrupts Development of Binaural Selectivity in Auditory Midbrain and Cortex. Neuron 65(5): 718-731.

29. Rae Lynn J Lamminen, Daniel Houlihan (2015) A Brief Overview of Amblyaudia. Health 7: 927-933.

30. Bilger RC, Nuetzel JM, Rabinowitz WM, Rzeczkowski C (1984) Standardization of a test of speech perception in AWM: Adults with Normal/Mild-Moderate Hearing loss noise. J Speech Hear Res 27(1): $32-48$.

31. RM Hurley (1999) Onset of Auditory Deprivation. Journal of the American Academy of Audiology. 10: 529-534.

32. Millman RE, Mattys SL (2017) Auditory Verbal Working Memory as a Predictor of Speech Perception in Modulated Maskers in Listeners with Normal Hearing. Journal of Speech, Language, and Hearing Research 60(5): 1236-1245. 


\section{Global Journal of Otolaryngology}

33. Barret DJ, Pilling M (2017) Evaluating the Precision of Auditory Sensory Memory as an Index of Intrusion in Tinnitus. Ear Hear 38(2): 262-265.

34. Liebel SW, Nelson JM (2017) Auditory and Visual Working Memory in College Students with Attention Deficit/Hyperactivity Disorder and/ or Learning disabilities. Archives of Clinical Neuropsychology 32(8): 980-991.

35. Miller CW, Stewart EK, Wu YK, Bishop C, Bentler RA, et al. (2017) Working Memory and Speech Recognition in Noise Under Ecologically Relevant Listening Conditions: Effects of Visual Cues and Noise Type 60(8): 2310-2320.

36. Fullgrade C, Rosen S (2016) On the (Un) importance of Working Memory in Speech-in-Noise Processing for Listeners with Normal Hearing Thresholds. Frontiers in Psychology 7: 1268.

37. Zekveld AA, Rudner M, Johnsrude IS, Rönnberg J (2013) The effects of working memory capacity and semantic cues on the intelligibility of speech in noise. The Journal of Acoustic Society of America 134(3): $2225-2234$

38. Jos JE (2015) Auditory Temporal Processing and its Disorders. United Kingdom: Oxford University Press, USA.

39. Baddeley AD (1986) Working Memory. Oxford Psychology Series No 11. Oxford: Clarendon Press.

40. Bamiou D, Musiek F, Luxon L (2001) Aetiology and clinical presentations of auditory processing disorders review. Arch Dis Child 85(5): 361-365.

41. Xu H, Kotak VC, Sanes DH (2007) Conductive hearing loss disrupts synaptic and spike adaptation in developing auditory cortex. Journal of Neuroscience 27(35): 9417-9426.

42. Albert B Blankenship (1938) Memory Span: A Review of the Literature. The psychological bulletin 35(1): 2-3.

43. American National Standards Institute [ANSI] (2003) American National Specification of Hearing Aid Characteristics. New York: Acoustical Society.

44. American Speech-Language-Hearing Association Task Force on Central Auditory Processing Consensus Development (1996) Central auditory processing: Current status of research and implications for clinical practice. American Journal of Audiology 5(2): 41-54.

45. Arlinger S, Lunner T, Lyxell B, Pichora-Fuller K (2009) The emergence of cognitive hearing science. Scand J Psychol 50(5): 371-384.

46. Ashcraft MA, Klein R (2011) Attention In: Levitin DJ Foundations of Cognitive Psychology-Core Readings $2^{\text {nd }}$ edn Boston: Allyn and Bacon.

47. Baddeley A (2000) The episodic buffer: a new component of working memory? Trends Cognitive Sciences 4(11): 417-423.

48. Baddeley AD, Hitch G (1974) Working memory. In: Bower GH, editor The Psychology of Learning and Motivation New York.

49. Baddeley AD, Scott D (1971) Short-term forgetting in the absence of proactive inhibition. Quarterly Journal Experimental Psychology 23: 275-283.

50. Baddeley AD, Warrington EK (1970) Amnesia and the distinction between long- and short-term memory. Journal of Verbal Learning and Verbal Behavior 9(2): 176-189.

51. Bayat A, Farhadi M, Emamdjomeh H, Saki N, Mirmomeni G, et al. (2010) Effect of conductive hearing loss on central auditory function. Braz J Otorhinolaryngol 83(2): 137-141.

52. Beck DL (2009) Multitasking, cognition, and cell phone distractions.
53. Beck DL (2010) Cognition and audition - Introductory concepts. Hearing Professional 59(4): 23-25.

54. Beck DL, Flexer C (2011) Listening Is Where Hearing Meets Brain in Children and Adult. Hearing Review p.30-35.

55. Esser J, Koelewijn T, Zekveld AA, Kramer SE, Festen JM (2013) How linguistic closure and verbal working memory relate to speech recognition in noise-A review. Trends Amplif 17(2): 75-93.

56. Boothroyd A (1997) Auditory development of the hearing child. Scand Audiol Suppl 46: 9-16.

57. Boudreau D, Costanza-Smith A (2011) Assessment and Treatment of Working Memory Deficits in School-Age Children: The Role of the Speech-Language Pathologist. Lang Speech Hear Serv Sch 42(2): 152166

58. Cole EB, Flexer C (2011) Children with Hearing Loss: Developing Listening and Talking, Birth to Six. $2^{\text {nd }}$ edn San Diego: Plural Publishing, USA.

59. Cowan N (2005) Working memory capacity. Hove, East Sussex, UK: Psychology Press, UK.

60. Cowan N (2010) The Magical Mystery Four: How is Working Memory Capacity Limited, and Why? Curr Dir Psychol Sci 19(1): 51-57.

61. Cole EB, Flexer C, (2011) Children with Hearing Loss: Developing Listening and Talking, Birth to Six. $2^{\text {nd }}$ edn, San Diego: Plural Publishing, USA.

62. Cowan N (2005) Working memory capacity. Hove, East Sussex, UK: Psychology Press.

63. Cowan N (2010) The Magical Mystery Four: How is Working Memory Capacity Limited, and Why? Current Directions in Psychological Science 19(1): 51-57.

64. Cruickshanks KJ, Tweed TS, Wiley TL, Klein BEK, Klein R, et al. (2003) The 5-year incidence and progression of hearing loss: the epidemiology of hearing loss study. Archives of Otolaryngology. Head and Neck Surgery 129(10): 1041-1046.

65. Dehaene S (2009) Reading in the Brain: The Science and Evolution of a Human Invention. New York: Penguin, pp. 400.

66. Desjardins JL, Doherty KA (2009) Do experienced hearing aid users know how to use their hearing aids correctly? American Journal of Audiology 18(1): 69-76.

67. Desjardins JL, Doherty KA (2013) Age-related changes in listening effort for various types of masker noises. Ear Hear 34(3): 261-272.

68. Doidge N (2007) The BRAIN That Changes Itself. Penguin Books, London, UK.

69. Ferguson MA, Henshaw H (2015) Auditory training can improve working memory, attention, and communication in adverse conditions for adults with hearing loss. Frontiers in Psychology 6: 556.

70. Chermak GD, Hall JW, Musiek FE (1999) Differential Diagnosis and Management of Central Auditory Processing Disorder and Attention Deficit Hyperactivity Disorder. Journal of American Academy of Audiology 10(6): 289-303.

71. Gladwell M (2008) Outliers: The Story of Success. New York: Little, Brown and Co.

72. Greenhouse SW, Geisser S (1959) On methods in the analysis of profile data. Psychometrika 24(2): 95-112.

73. Guenther RK Memory In: Levitin DJ (2011) Foundations of Cognitive Psychology - Core Readings. $2^{\text {nd }}$ edn. ISBN 9780205711475. Boston: Allyn and Bacon. 


\section{Global Journal of Otolaryngology}

74. Hart B, Risley TR (1999) The Social World of Children Learning to Talk. Baltimore: Brookes.

75. Jirsa R (2002) Clinical efficacy of electro physiologic measures in auditory processing disorders management programs. Seminars in Hearing 23(4): 349-356.

76. Joint Committee on Infant Hearing (2007) Principles and guidelines for early hearing detection and intervention programs. Pediatrics 102(4): 893-921.

77. Kronenberger WG, Pisoni DB, Henning SC, Colson BG, Hazzard LM (2011) Working memory Training for Children with Cochlear Implants-A Pilot Study. Journal of Speech, Language and Hearing Research 54(4): 1182-1196.

78. Levitin DJ (2006) This Is Your Brain on Music. New York: Penguin.

79. Lin F (2011) Hearing loss and cognition among older adults in the United States. J Gerontol A Biol Sci Med Sci Series A 66(10: 1131-1136.

80. Masters M, Stecker N \& Katz J (1998) Central auditory processing disorders: Mostly management. Boston, MA: Allyn and Bacon.

81. Matthew TH, Kevin WG, Kevin JB, Jeffery ML, Adrianne B (2005) WAIS Digit Span-Based Indicators of Malingered Neurocognitive Dysfunction Classification Accuracy in Traumatic Brain Injury. Assessment 12(4): 429-444.

82. Among Adults with Hearing Loss. Journal of Speech, Language and Hearing Research 25: 1-11.

83. Moore DR (2007) Auditory processing disorders: Acquisition and treatment. Journal of Communication Disorders 40(4): 295-304.

84. Moucha R, Kilgard MP (2006) Cortical plasticity and rehabilitation. Progress in Brain Research 157: 111-122.

85. Mulrow CD, Aguilar C, Endicott JE, Tuley MR, Velez R, et al. (1990) Quality-of-life changes and hearing impairment: a randomized trial Annals of Internal Medicine 113(3): 188-194.

86. Murphy DR, Mc Dowd JM, Wilcox KA (1999) Inhibition and aging: similarities between younger and older adults as revealed by the processing of unattended auditory information. Psychology and Aging 14(1): 44-59.
87. Musiek FE, Berge B (1998) A neuroscience view of auditory training stimulation and central auditory processing disorders. In MG Masters, NA Stecker, \& J Katz (Eds.) Central Auditory Processing Disorders. Mostly management. Boston MA: Allyn \& Bacon.

88. National Institute on Deafness and Other Communication Disorders (2012) Hearing, Ear Infections, and Deafness.

89. Rabbitt PMA (1990) Age, IQ and awareness of errors. Ergonomics 33(10-11): 1291-1305

90. Pugh K, Sandak R, Frost SJ (2006) Neurobiological investigations of skilled and impaired reading. Handbook of Early Literacy Research 2 New York: Guilford.

91. Robbins AM, Koch DB, Osberger MJ, Zimmerman-Philips S, KishonRabin L (2004) Effect of age at cochlear implantation on auditory skill development in infants and toddlers. Archives of Otolaryngology Head and Neck Surgery 130(5): 570-574.

92. Rönnberg J (2003) Cognition in the hearing impaired and deaf as a bridge between signal and dialogue: a framework and a model. International Journal of Audiology 42: S68-S76.

93. Rönnberg J, Rudner M, Foo C, Lunner T (2008) Cognition counts: A working memory system for ease of language understanding (ELU). International Journal of Audiology 47: S99-S105.

94. SA Gelfand (1995) Long-Term Recovery and No Recovery from the Auditory Deprivation Effect with Binaural Amplification: Six Cases. Journal of American Academy of Audiology 6(2): 141-149.

95. Smaldino JJ, Crandell CC (2000) Classroom amplification technology: theory and practice. Language, Speech and Hearing Services in Schools pp. 371-375.

96. Van Boxtel MP, Van Beijsterveldt CE, Houx PJ, Anteunis LJ, Metsemakers JF, et al. (2000) Mild hearing impairment can reduce verbal memory performance in a healthy adult population. Journal of Clinical and Experimental Neuropsychology 22(1): 147-154.

97. Whitelaw GW, Yuskow K (2005) Neuromaturation and neuroplasticity. In Parthasarathy TK (Ed.) Introduction to auditory processing and its disorders Mahwah, NJ Lawrence Erlbaum pp. 21-38.

\section{Your next submission with Juniper Publishers will reach you the below assets}

- Quality Editorial service

- Swift Peer Review

- Reprints availability

- E-prints Service

- Manuscript Podcast for convenient understanding

- Global attainment for your research

- Manuscript accessibility in different formats

( Pdf, E-pub, Full Text, Audio)

- Unceasing customer service

Track the below URL for one-step submission

https://juniperpublishers.com/online-submission.php 